\title{
The relation between dilatancy, effective stress and dispersive pressure in granular avalanches
}

\author{
Perry Bartelt ${ }^{1} \cdot$ Othmar Buser $^{1}$
}

Received: 30 September 2015/ Accepted: 22 April 2016/Published online: 21 May 2016

(C) Springer-Verlag Berlin Heidelberg 2016

\begin{abstract}
Here we investigate three long-standing principles of granular mechanics and avalanche science: dilatancy, effective stress and dispersive pressure. We first show how the three principles are mechanically interrelated: Shearing of a particle ensemble creates a mechanical energy flux associated with random particle movements (scattering). Because the particle scattering is inhibited at the basal boundary, there is a spontaneous rise in the center of mass of the particle ensemble (dilatancy). This rise is connected to a change in potential energy. When the center of mass rises, there is a corresponding reaction at the base of the flow that is coupled to the vertical acceleration of the ensemble. This inertial stress is the dispersive pressure. Dilatancy is therefore not well connected to effectivestress-type relations, rather the energy fluxes describing the configurational changes of the particle ensemble. The strict application of energy principles has far-reaching implications for the modeling of avalanches and debris flows and other dangerous geophysical hazards.
\end{abstract}

Keywords Avalanches · Bagnold · Cohesion · Dilatancy · Dispersive pressure $\cdot$ Density $\cdot$ Effective stress $\cdot$ Flow regime $\cdot$ Granular mechanics $\cdot$ Jerk $\cdot$ Reynolds $\cdot$ Terzaghi

Perry Bartelt

bartelt@slf.ch

1 WSL Institute for Snow and Avalanche Research SLF, Davos Dorf, Switzerland

\section{Introduction}

Three central concepts of granular mechanics are dilatancy, effective stress and dispersive pressure. All three principles are applied to describe the mechanics of hazardous, gravitationally driven mass movements, particularly snow and rock avalanches and debris flows.

\subsection{Reynolds and dilatancy}

Dilatancy was first introduced by Reynolds [26] to explain the increase in volume when a material composed of rigid particles is sheared. Reynolds, in fact, believed this was the distinctive feature of granular materials, making granular mechanics unique and set apart from the field of continuum mechanics. However, in 1886 Reynolds wrote “...but subsequent consideration revealed the striking fact, that a medium composed of grains of any possible shape possessed this property of dilatancy, as long as one important condition was satisfied. This condition is, that the medium should be continuous, infinite in extent, or that the grains at the boundary should be held as to prevent a rearrangement commencing.", see [25], page 219. Reynolds was therefore aware that any physical description of dilatancy was related to the nature of the applied shearing relative to the boundary conditions defining the geometry of the dilation.

\subsection{Terzaghi and effective stress}

Terzaghi's effective stress concept appeared some 40 years later $[10,29]$ and has become a cornerstone of granular mechanics. Terzaghi modified Coulombs well-accepted equation combining shear stress $\tau$, internal friction angle $\phi$ and total stress $\sigma$ 


$$
\tau=\sigma \tan (\phi)
$$

to include the pore water pressure $p$

$$
\tau=(\sigma-p) \tan (\phi)=\sigma^{\prime} \tan (\phi),
$$

calling the new quantity $\sigma^{\prime}=\sigma-p$ the effective stress. The effective stress models the average normal intergranular contact force per unit area as the difference $\sigma-p$ describes the buoyant weight of the solid phase. Effective stress concepts have been invoked to model the shearing at the basal running surface of debris flows (e.g., [9, 14]) and dry snow avalanches (e.g., [12, 23]).

\subsection{Bagnold and dispersive pressure}

The third concept, dispersive pressure, was introduced still another 30 years later by Bagnold [1]. Bagnold calculated the pressure that acts to disperse grain layers within a fluid saturated granular medium and found that the pressure is proportional to the square of the shear rate. Bagnold uses the dispersive stress concept to predict the velocity of several experimental avalanches composed of dry sand, see [1], page 62. He postulated that this dispersive pressure was, " ...of such a magnitude that an appreciable part of the moving grains is in equilibrium between it and the force of gravity...", see [1], page 50. Physically this statement is tantamount to replacing the pore water pressure term in the effective stress Eq. (2) with the dispersive pressure. Thus, the effective stress concept was implicitly seen to be valid for both water saturated and dry granular flows. In both cases the normal stress is reduced, although by different pressures, either by the pore pressure or by the dispersive pressure. Bagnold's comparison between the experimental results and dispersive model calculations was rather poor, at least for today's standards. This give credence to recent criticisms of Bagnold's experiments [11]. However, it does not alter the fact that shearing increases the pressure-at constant volume.

\subsection{Bagnold, Terzaghi and Reynolds}

Bagnold was not only aware of the work of Terzahgi (see [1], page 62) but also of the much earlier work of Reynolds. Bagnold invokes Reynolds concept of dilatancy to explain how the dispersive pressure is responsible for a change of shear stress. He writes: “...a shear stress causes a tendency to dilate. If this is resisted by a compressive stress the ratio of shear stress-compressive stress is near unity. But if the dilation is allowed to proceed to the static limit, the ratio falls to that given by the tangent of the angle of repose. It will be shown that these relations appear to have their counterparts in the dynamic case of continuing shear strain when the limit of static dilation has been far exceeded and the grain mass has become a dispersion." Thus, in Bagnold, we find a combination of all three principles: shearing causes a dispersive pressure, that causes a dilation, that causes (in the dynamic case) a change in the normal stress, linked at static equilibrium $(p=0)$ to the angle of repose. The dynamic case being defined when "the limit of static dilation" is exceeded; moreover, when the dispersive pressure is strong enough $(p>0)$ to reduce the compressive stress (weight or static dilation) of the flow.

\subsection{Avalanche modeling}

Most of todays avalanche and debris flow models are based on Savage-Hutter-type equations that assume a constant flow density [28]. In the terminology of Bagnold, they always remain at the limit of static dilation. This is the fundamental problem in avalanche and debris flow physics, that is only recently earning attention [6, 14]. At present, dispersive pressures are used within the framework of Terzaghi's effective stress principle, but are not directly associated with the energy fluxes required to change the configuration of the flow volume. Even sedimentation and re-supension of particles induce a change in the position of the center of mass [17]. Recent models that invoke dilatancy as a fundamental physical property of avalanches [14] short-circuit the mechanics of dilatancy by using effective stress principles without consideration of the actual dispersion, and therefore randomness, not only of the motion, but also position of granules. This limits the application of existing models to specific flow regimes; it also prevents a systematic calibration of model parameters because the many flow states exceeding the limit of static dilation are ignored. For example, how boundary conditions-including both terrain roughness and entrainment processes-influence the flow configuration cannot be rationally explored and therefore cannot be included in a detailed hazard analysis.

\subsection{Motivation}

In this paper we investigate the energy fluxes associated with dilatancy. There are two: (1) the kinetic energy associated with random particle motions and (2) the potential energy associated with random particle positions. The source of these two energies was identified by Reynolds, Terzaghi and Bagnold: shearing, or more specifically, the work done by shear. We show why the boundary conditions play an important role in partitioning of the random, dispersive kinetic and potential energies. These considerations allow us to calculate Bagnold's flow states that are beyond the limit of static dilation without consideration of the minutiae of particle interactions. Above 
all, we identify the physical and mathematical properties of the dispersive pressure.

\section{Dilatancy and dispersive pressure}

\subsection{Bagnold's limit of static dilation: the co-volume}

To understand the mechanics of dilatancy we begin by dividing an avalanche into representative volumes $V$ (Fig. 1). The volumes are defined by the (constant) basal area $A$ and flow height $h$, measured from the bottom of the avalanche. The center of mass is located at height $k$. The volumes are fixed to a particular location (Eulerian formulation) and mass flows through the volume with mean velocities $\mathbf{u}=(u, v)^{\mathrm{T}}$. The velocity vectors are defined in the slope-parallel direction. The volume $V$ contains the solid particle mass $M$. The mass is defined per unit area $A$. The configuration of the particle mass within the avalanche core can vary (Fig. 3). This gives rise to different densities in the core. At the static limit, the particle mass is collapsed into a solid volume with height $h_{0}$ measured with reference to the density of a random packing density $\rho_{0}$ :

$M=\rho_{0} h_{0}$.

Because the height $h_{0}$ represents the height of a dry deposition pile of particles, it is given a special designation, the co-volume height. The center of mass of the co-volume is located at $k_{0}$. For example, we could assume that the particle mass is distributed uniformly over the flow volume, in which case,

$M=\rho h$.

The heights $h_{0}$ and $h$ represent two different flow configurations that the avalanche core may assume with the same mass. The height $h_{0}$ is typically encountered when the avalanche has settled in the deposition zone, the random packing density being close to the deposition density of the particles. That is, it is encountered when the avalanche core is in static equilibrium. A mechanical treatment of dilatancy requires that we understand how under the action of shearing the volume expands from the co-volume height $h_{0}$ to the flow height $h$.

\subsection{Random dispersive and configurational energies}

The two configurations in Fig. 2 are associated with two mechanical energies, see Fig. 3. The first is the random kinetic energy $R_{\mathrm{K}}$ that associated with all particle movements different from the mean velocity of the flow (the socalled granular temperature). Shearing of the particle ensemble causes the particles to scatter and have some velocity component in the slope-perpendicular direction, that is, different from the mean downslope direction of the flow $\mathbf{u}$. The total kinetic energy associated with the slopeperpendicular movement and all fluctuations around the mean velocities in the slope-parallel directions is contained in $R_{\mathrm{K}}$. The scattering of the particles necessarily implies some change in the particle positions and change in ensemble configuration. We define the quantity $R_{\mathrm{V}}$ as the configurational energy associated with the potential energy of the center of mass of the particles, see Figs 2 and 3,

$$
R_{\mathrm{V}}=\operatorname{Mg}\left(k-k_{0}\right) \text {. }
$$

When $R_{\mathrm{V}}=0$, we are at the limit of static dilation, i.e., the co-volume. Note that $R_{\mathrm{V}}$ and the potential energy differ by the potential energy of the co-volume. When $R_{\mathrm{V}}>0$ we have a change in the particle configuration. We consider $R_{\mathrm{K}}$ and $R_{\mathrm{V}}$ to be evenly distributed over the volume $V$. This assumption is equivalent to depth-averaging.

\subsection{Production of dispersive and configurational energies}

The energies $R_{\mathrm{K}}$ and $R_{\mathrm{V}}$ cannot be studied without considering their origin. Bagnold recognized that shearing produces a scattering. However, the shearing energy always produces the configurational energy $R_{\mathrm{V}}$ at the same time. It is the interaction of the random moving particles with the boundary that causes the volume expansion.

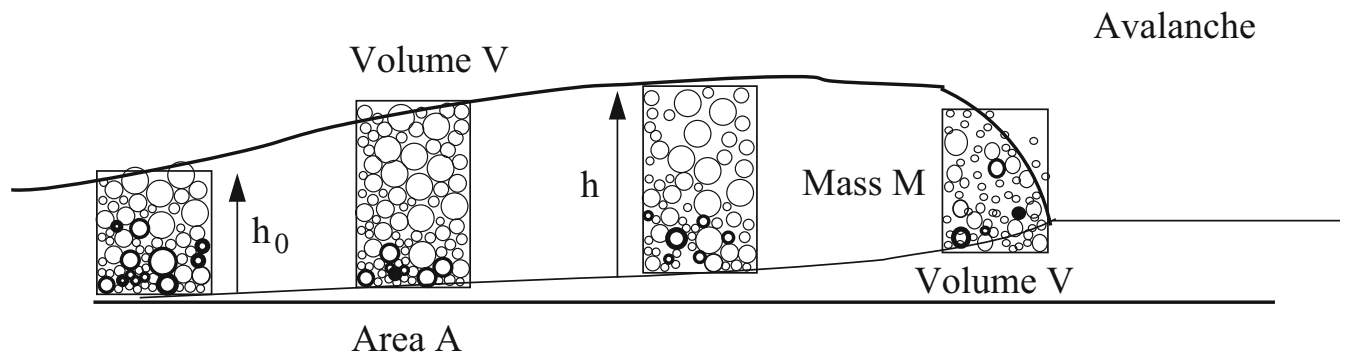

Fig. 1 A granular avalanche consists of flow volumes $V$. Each volume is defined by the fixed basal area $A$ and the flow height $h$. Each volume is considered a particle ensemble with mass $M$. The location of the highest particle defines the location of the top surface of the volume. The density of each volume is therefore $\rho V=M$. The density of each volume can vary in the streamwise flow direction 


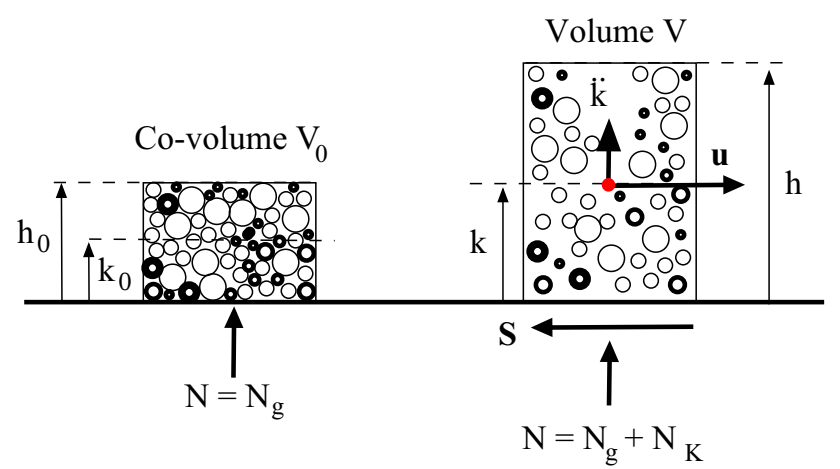

Fig. 2 The co-volume $V_{0}$ represents Bagnold's limit of static dilation. The height of the co-volume is $h_{0}$ and the center of mass is located at $k_{0}$. The co-volume characterizes the static equilibrium of the particle ensemble. The basal normal stress is given by the weight $N_{\mathrm{g}}$. Under shearing the co-volume expands to the height $h$. When the co-volume dilates, a dispersive reaction $N_{\mathrm{K}}$ is produced by the acceleration $\ddot{k}$ of the center of mass. The dispersive pressure exists only when there is a corresponding acceleration of the center of mass. If the center of mass does not move, $N_{\mathrm{K}}=0$

However, the volume expansion is only in one direction normal to the basal surface because of the boundaries. In fact with no boundaries, there would be a volume change, but no change in $R_{\mathrm{V}}$, merely a random scattering of particles. We define the production of $R_{\mathrm{K}}$ and $R_{\mathrm{V}}$ in the volume $V$ to be $\dot{P}_{\mathrm{K}}$ (change in random kinetic energy) and $\dot{P}_{\mathrm{V}}$ (change in configuration energy). Physically, $\dot{P}_{\mathrm{K}}$ and $\dot{P}_{\mathrm{V}}$ must coexist and have the property

$\dot{P}_{\mathrm{V}}=\gamma \dot{P} \quad$ and $\quad \dot{P}_{\mathrm{K}}=(1-\gamma) \dot{P}$,

where $\dot{P}$ is the total production

$\dot{P}=\dot{P}_{\mathrm{K}}+\dot{P}_{\mathrm{V}}$.
That is, there exists some partitioning parameter $\gamma$ that defines how much of the random scattering energy is transformed into dilatancy [7, 8]. Dilatancy being defined as a change in gravitational potential energy $R_{\mathrm{V}}$. The dilatancy parameter $\gamma$ is physically constrained between the values $0<\gamma<1$. Values $\gamma=0$ or $\gamma=1$ are physically impossible because $R_{\mathrm{V}}$ and $R_{\mathrm{K}}$ coexist. The dilatancy parameter depends on the mechanical and geometric properties of the particles, but also on the hardness of the basal boundary. Balance equations for the free mechanical energy can be written,

$$
\begin{aligned}
& \frac{D\left(R_{\mathrm{K}} h\right)}{D t}=\dot{P}_{\mathrm{K}} h, \\
& \frac{D\left(R_{\mathrm{V}} h\right)}{D t}=\dot{P}_{\mathrm{V}} h
\end{aligned}
$$

and

$$
\frac{D(R h)}{D t}=\dot{P} h=\dot{P}_{\mathrm{K}} h+\dot{P}_{\mathrm{V}} h .
$$

These equations are written using the material derivative notation to indicate that we must also consider the convective transport of $R_{\mathrm{K}}$ and $R_{\mathrm{V}}$.

\subsection{Shearing, the source of Reynolds' dilatancy}

Shearing is the immediate source of the energy needed to change the configuration of the avalanche and expand the volume $V$. The shear work rate $\dot{W}_{f}$ is

$$
\dot{W}_{f}=\mathbf{S} \cdot \mathbf{u}
$$

where $\mathbf{S}$ is the shear stress. The work rate $\dot{W}_{f}$ represents the total work done per unit time and area, that is, the depth-

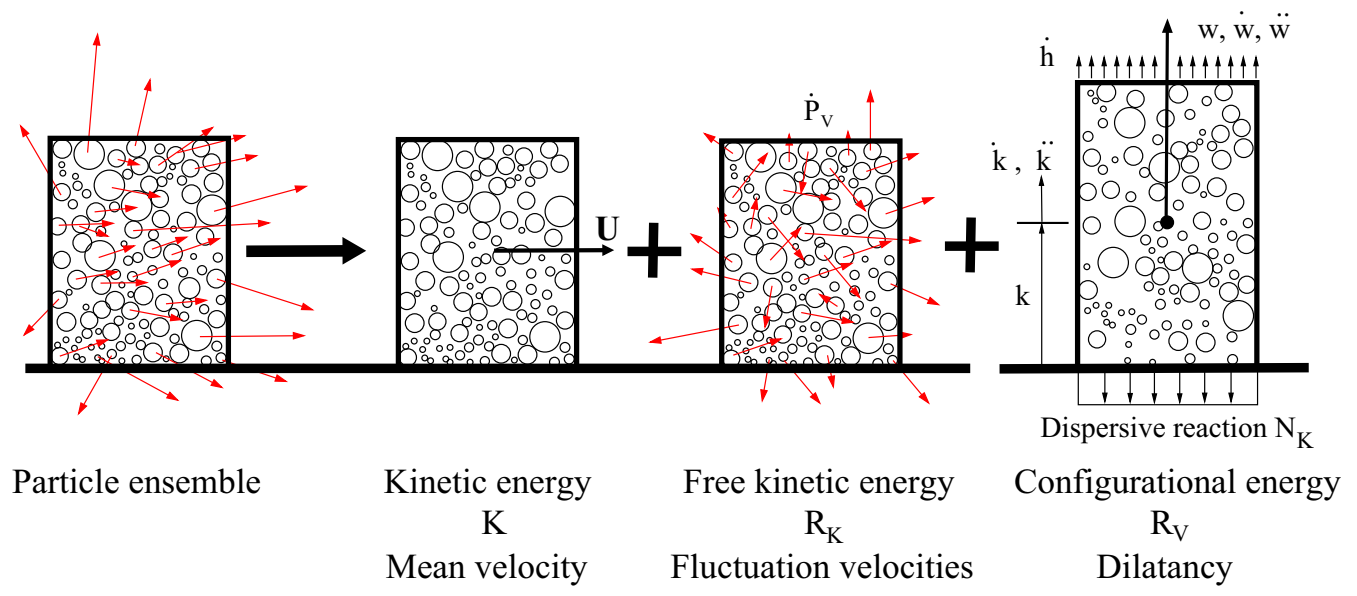

Fig. 3 Energies of the granular ensemble. Particle mass is moving through the volume $V$ with mean velocity $\mathbf{u}$. This defines the translational kinetic energy $K$ in the slope-parallel direction. The random kinetic energy $R_{\mathrm{K}}$ is associated with the particle velocity fluctuations and represents all kinetic energy not in $K$. This energy is transformed into potential energy $R_{\mathrm{V}}$ because of the basal boundary. The sum of $R_{\mathrm{K}}$ and $R_{\mathrm{V}}$ defines the free mechanical energy of the flow 
averaged work $\left(\mathrm{W} \mathrm{m}^{-2}\right)$. Bagnold, however, was very aware that shearing dissipates mechanical energy to heat energy $E$ at the rate $\dot{Q}$ but also is responsible for the production of free energy $\dot{P}$,

$\dot{W}_{f}=\dot{Q} h+\dot{P} h$.

Different relations can be used to separate the dissipation from the production of free mechanical energy. In snow avalanche models, the following splitting is applied $[2,30]$

$$
\begin{aligned}
& \frac{D(R h)}{D t}=\dot{P} h=\alpha \dot{W}_{f}-\beta R_{\mathrm{K}} h, \\
& \frac{D(E h)}{D t}=\dot{Q} h=(1-\alpha) \dot{W}_{f}+\beta R_{\mathrm{K}} h .
\end{aligned}
$$

The parameter $\alpha$ is the dispersion parameter, defining the partitioning of the frictional work rate into the production of free mechanical energy $\dot{P}$ and heat energy $\dot{Q}$. Similar to the dilatancy parameter $\gamma$, the range of the dispersion parameter is theoretically limited to values 0 $<\alpha<1$. The parameter $\beta$ defines the dissipation of free kinetic energy of the granular solid by collisions, rubbing, abrasion, etc. The value $\beta=0$ is reserved for purely elastic systems not found in nature.

\subsection{Dispersive acceleration, not dispersive pressure}

If the particle interactions generate pressures to change the location of the center of mass, there must be a corresponding reactive pressure at the basal boundary as shown in Figs. 2 and 3. This pressure is given by the mass $M$ in the avalanche core and the acceleration $\ddot{k}$ (Newton),

$N_{\mathrm{K}}=M \ddot{k}$.

The speed the center of mass is given by $\dot{k}=w$, see Fig. 2. The total reaction at the basal boundary $N$ is the sum of the weight $N_{\mathrm{g}}$

$N_{\mathrm{g}}=M g_{z}$

and $N_{\mathrm{K}}$,

$N=N_{\mathrm{g}}+N_{\mathrm{K}}$.

The gravity component in the slope-perpendicular direction is denoted $g_{z}$. The sum of the accelerations $g_{z}$ and $\ddot{k}$ is denoted $g^{\prime}$. Because we include the pressure $N_{\mathrm{K}}$, the pressure is no longer hydrostatic. $N_{\mathrm{K}}$ can be negative if the center of mass is falling. Basal pressure measurements cannot distinguish between a change in mass $M$ or a change in the location of the center of mass $k$ because the total normal force $N$ is the sum of $N_{\mathrm{g}}$ and $N_{\mathrm{K}}$ [24]. It is therefore difficult to determine the pressure $N_{\mathrm{K}}$ experimentally. A connection to experimental measurements can nonetheless be made by noting that the time rate of change of the normal pressure $\dot{N}$ is (from Eq. 15)

$\dot{N}=M \dddot{k}$

when the mass is constant in the volume $V$. Because gravitational acceleration $g_{z}$ is constant, it disappears from the time derivative. The quantity $\dddot{k}$ is the jerk, the change in acceleration, the avalanche experiences from the shear interaction with the boundary.

\subsection{Change in flow configuration}

The problem now is to relate the configurational energy production per volume $\dot{P}_{\mathrm{V}}$ to the change in the location of the center of mass $k$. We emphasize that $\dot{P}_{\mathrm{V}}$ is simply some fraction of the shear work rate $\dot{W}_{f}$ that is transformed into potential energy. The work done by $\dot{P}_{\mathrm{V}}$ is used to change the volume $V$ of the avalanche. Let $W_{\mathrm{V}}$ be the work per unit area done by the particles at the basal boundary. This work is the product of the normal force $N$ and the change in volume,

$$
W_{\mathrm{V}}=N k=\int_{0}^{t} \dot{P}_{\mathrm{V}}(t) h(t) \mathrm{d} t=\gamma \int_{0}^{t} \dot{P}(t) h(t) \mathrm{d} t .
$$

This integral relation connects the dilatancy directly to the shear power. It explicitly assumes a hard basal boundary and the direction of the dilation, $k$. Because the product of the production of free mechanical energy $\dot{P}$ (per volume) and flow height $h$ is known, it is more convenient to write the integral equation as a differential equation. Moreover,

$$
\dot{W}_{\mathrm{V}}=\frac{\mathrm{d}(N k)}{\mathrm{d} t}=\dot{P}_{\mathrm{V}} h
$$

or,

$$
\dot{N}+N \frac{\dot{k}}{k}=2 \gamma \dot{P}=2 \dot{P}_{\mathrm{V}},
$$

when we know the relation between the height $h$ and center of mass location $k, k=h / 2$ (homogeneous mass distribution). By substitution of Eqs. 15-18

$M \dddot{k}+M\left[g_{z}+\ddot{k}\right] \frac{\dot{k}}{k}=2 \dot{P}_{\mathrm{V}}$.

This equation can be conveniently written into a series of three first-order differential equations, namely

$$
\begin{aligned}
& \frac{D k}{D t}=w(t), \\
& \frac{D(M w)}{D t}=N_{\mathrm{K}}(t),
\end{aligned}
$$


Fig. 4 Dilation of a particle ensemble with a free upper surface and a hard basal surface. a The work done by shear. A shear deformation is applied to a particle ensemble at time $t_{0}$. The shear deformation is constant at time $t_{1}$. b Free mechanical energy $R$. The free energy increase from zero and is constant after time $t_{1}$. $\mathbf{c}$ The production of free mechanical energy $\dot{P}=\dot{P}_{\mathrm{K}}+\dot{P}_{\mathrm{V}}$. d The reaction jerk at the basal boundary $\dot{N}_{\mathrm{K}}$. e The dispersive reaction $N_{\mathrm{K}}$ and the total normal stress $N$. f The volume has expanded from the co-volume height $h_{0}$ to the height $h$. The dispersive pressure exists only during the change from height $h_{0}$ to $h$

$\frac{1}{2} \frac{D N_{\mathrm{K}}}{D t}+\rho(t)\left[g_{z}+\ddot{k}(t)\right] w(t)=\dot{P}_{\mathrm{V}}(t)$

These first-order equations allow us to include the advective transport of $k$ (avalanche height), $M w$ (momentum in the $z$-direction) and $N_{\mathrm{K}}$. Moreover, the jerk associated with the mass transport can be considered in the depth-averaged models. This is of great practical significance, because when a volume dilates, the z-velocity and $\mathrm{z}$-acceleration of the "blow-out" or "eruption" must be transported forward with the mass. This is especially important for the formation of powder snow avalanches [5].

\section{Dilatancy and the fallacy of effective stress measures}

\subsection{Work-energy description of dilatancy}

The above description of dilatancy is based on work-energy mechanics and corresponds to both Reynolds' ideas of dilatancy and Bagnold's description of the effects of random dispersion. We can now describe the dilation as a six step process, see Fig. 4:

1. Shearing We apply a shearing to a particle ensemble. At time $t=0$, the volume is at rest; at $t=t_{0}$ the shear is applied such that the shear work rate or shear power increases linearly. At time $t=t_{1}$, the shear work rate is constant, see Fig. 4a. Shearing of the granular ensemble creates a random scattering of particles, not only heat. The kinetic energy associated with the random scattering is designated $R_{\mathrm{K}}$, and the production is denoted $\dot{P}_{\mathrm{K}}$. The division of the shear work into heat and random kinetic energy is defined by the dispersion parameter $\alpha$.

2. Production of free mechanical energy Under the action of this time-dependent shearing, the production of free mechanical energy in the volume rises from zero to a nearly constant a value as shown in Fig. 4b. However, when the shear power becomes constant, the production decreases to zero. At this state, the production from the constant shearing is in balance with the decay of
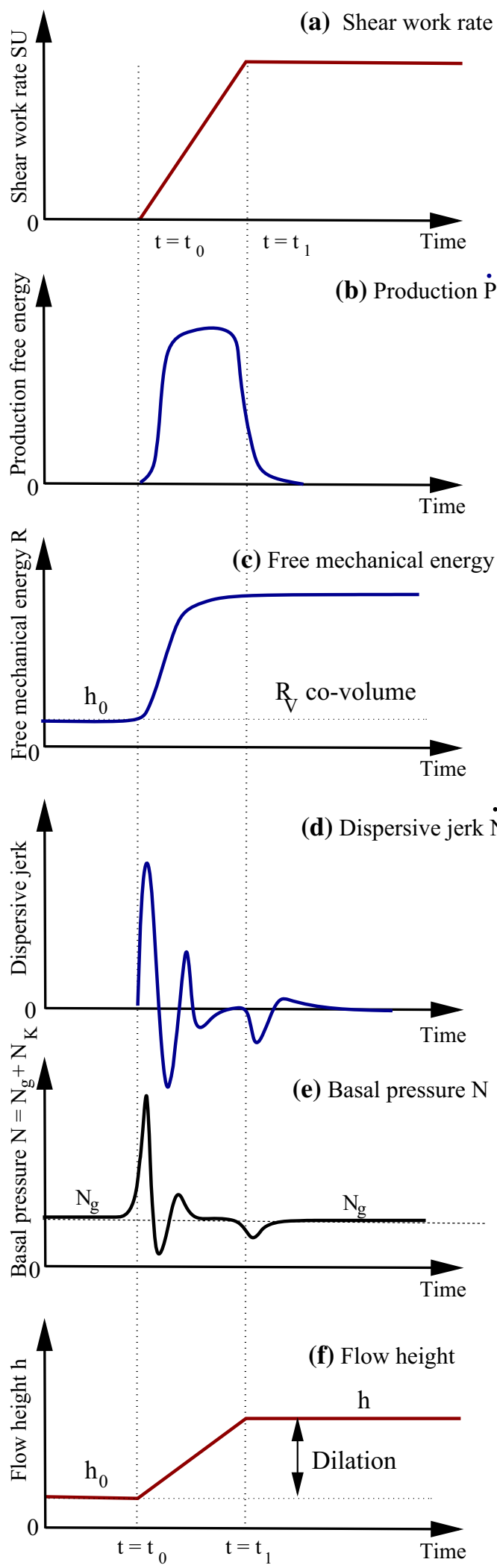

random energy. In this case, the net production is zero $\dot{P}=0$ and the free energy is constant $R=$ constant. This case is equivalent to a constant height (dilatancy). 
3. Free mechanical energy The free mechanical energy increases from the initial potential energy of the covolume to a constant value defined by the balance between the shear production and decay (parameter $\beta$ ) as shown in Fig. 4c. The constant value of free mechanical energy corresponds to the constant shear power.

4. Jerk, dispersive acceleration Because there is a hard basal boundary at the bottom of the avalanche, a part of the particle scattering is used to raise the center of mass and change the configuration of the particle ensemble. The center of mass is jerked upwards as shown in Fig. 4d. The flux of random kinetic energy $\dot{P}$ at the boundary is converted into the production of potential energy $\dot{P}_{\mathrm{V}}$. The dilatancy parameter $\gamma$ defines the part of the total production that remains as random kinetic energy $R_{\mathrm{K}}$ and the part simultaneously converted to potential energy $R_{\mathrm{V}}$. The change in the center of mass represents the mean dilation of the volume defined by the configurational energy $R_{\mathrm{V}}$. The jerk disappears when the shearing is constant.

5. The basal pressure At the beginning of the shearing the reactive pressure at the bottom is given by the weight of the particle ensemble. When the shear is applied at time $t=t_{0}$, the basal pressure at the bottom increases as the center of mass is accelerated upwards. At time $t=t_{1}$, the shear power is constant and the volume is sustained. The jerk disappears and the basal pressure returns to the weight of the particle ensemble as shown in Fig. 4e.

6. Dilatancy The height of the volume increases from the co-volume height $h_{0}$ to the dilated height $h$. Although the height of the ensemble has changed, the pressure at the bottom is unchanged. Only when the height is changing, there is a corresponding change in pressure (jerk).

\subsection{Stress- and energy-based descriptions of dilatancy are not equivalent}

There is a fundamental difference between stress-based and energy-based descriptions of dilatancy. Stress-based formulations postulate the existence of a dispersive pressure $p$ that not only changes the location of the center of mass when the ensemble is sheared, but also maintains the unchanging position of the center of mass when the shearing is constant. There is no stress measure that can fulfill both roles. There is undoubtedly a stress that changes the location of the center of mass; however, once the volume has dilated, this stress no longer acts and the system is in equilibrium, the reaction at the bottom of the avalanche returns to hydrostatic, see Fig. 4. The dispersive pressure is directly related to the change in shear work, which produces fluxes of random energies $\dot{P}_{\mathrm{K}} \neq 0$ and $\dot{P}_{\mathrm{V}} \neq 0$. When $\dot{P}=0$, the dispersive pressure disappears and the dilation is maintained by a constant random kinetic energy, $R_{\mathrm{K}}=$ constant. This energy is constant when the production by shearing is in balance with the decay caused by inelastic interactions. For an interesting discussion of the equilibrium/out-of-equilibrium role of dispersive pressure in inverse grading, see [18-20].

\subsection{Effective stress cannot be measured, only calculated}

The problem with effective stress is that it cannot be directly measured. It is impossible to measure the effective stress because it represents the mean stress acting on the solid phase, $N-p$. If such a stress exists, then there must be an equal and opposite stress, a reaction, at the basal boundary, see Fig. 5. This stress must be associated with the acceleration of the center of mass. Thus $N_{\mathrm{K}}$ is the dispersive pressure, but defined where it can be identified in experiments, as a reaction at the basal surface; moreover $N_{\mathrm{K}}=-p$. The mean total normal stress $N$ can be measured in chute experiments using force plates [24]. We emphasize that the volume dilation occurs in a field of constant acceleration (gravity) and therefore the dispersive acceleration can only be captured as a change in acceleration, or a jerk, at the basal boundary. When there is no change in

(a)

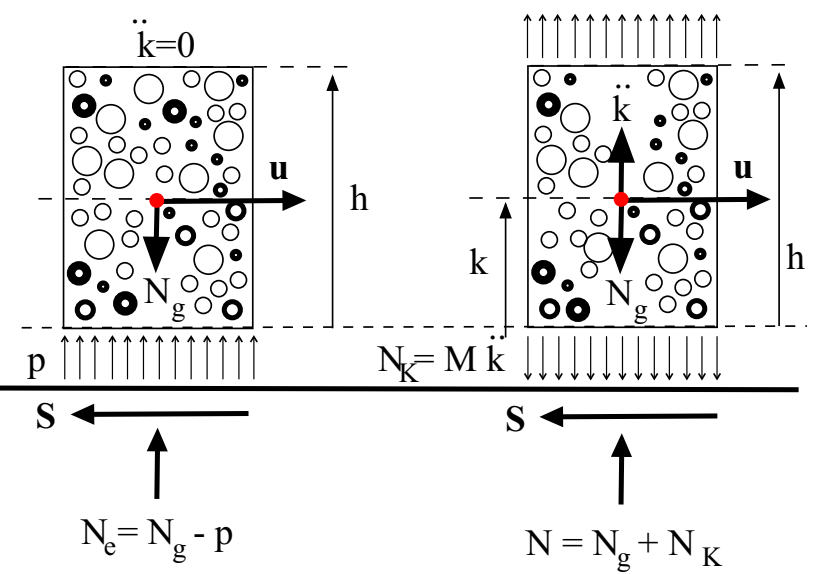

Fig. 5 The force balance at the base of the avalanche. a The effective stress concept introduces only the pressure $p$. There is no acceleration of the mass upwards, $\ddot{k}=0$ (in violation of Newtons law) and there is no equal and opposite reaction at the bottom (in violation of Newtons law). Effective stress principles are used without dilatancy. The force on the bottom is given by the effective pressure $N_{e}=N_{\mathrm{g}}-p$. b Energy-based approaches assume granule scattering induces an acceleration upwards $\ddot{k} \neq 0$. The upwards acceleration has a reaction at the bottom $N_{\mathrm{K}}$. The total force on the bottom is given by the sum of the weight $N_{\mathrm{g}}$ and the reaction to the dispersive acceleration $N_{\mathrm{K}}$ 
the center of mass, $N_{\mathrm{K}}=0$ and the mean pressure is $N=$ $N_{\mathrm{g}}$ (hydrostatic). Therefore, it is possible to distinguish between the weight component $N_{\mathrm{g}}$ and the dispersive reaction $N_{\mathrm{K}}$ associated with the change in the center of mass. It requires independent measurements of flow mass (flow height, density, etc.). Excess pressures that are measured in large-scale experiments [13, 22] or laboratory experiments $[15,16]$ can be better understood with unsteady descriptions of dilatancy and rapid changes in the location of the center of mass.

\section{Conclusions}

\subsection{Dilatancy and unsteady flow}

In real avalanches the shear power is hardly ever such that we have a steady state. In order to understand dilatancy, we must understand how we come from one steady state to another. Dilatancy is very much related to the unsteadiness of the flow because it describes the change in flow height. The steady flow state reflects a constant height and therefore isobaric conditions. Dilatancy requires departing from isobaric conditions in order to move the center of mass against gravity. This requires an input of random kinetic energy. The source of this energy is given by the shear power. This produces a change in acceleration of the center of mass, which is called a jerk. At steady state there is no jerk and therefore no change in flow height. Similar to thermodynamics, a change in height is directly related to a change in energy $(P V=n k T)$. The kinematics of dilatancy is independent of the constitutive relation for shear.

\subsection{Granular minutiae}

The dispersive pressure has often been related to the force imparted to the ground by a single particle collision. This force is related to the pre- and post-impact speed of the particle and therefore the coefficient of restitution describing the particle/ground impact, see Fig. 6a. The result of a multitude of particle impacts defines the collisional footprint and therefore the uplifting force or dispersive pressure. Here we adopt a different viewpoint: Instead of studying a single impact we ask what is the net result of the particle scattering and the interaction with the boundary? Our recent experience with the rockfall problem reveals that the outcome of an inelastic collision is poorly described by ideal restitution coefficients because they do not account for particle shape and complexity of the impact configuration [21]. If the result is an upward acceleration of the particle ensemble, then the mean pressure must be equal to the reaction of the inertial force, see Fig. $6 \mathrm{~b}$. We do not attempt to go from a single particle collision to (a)

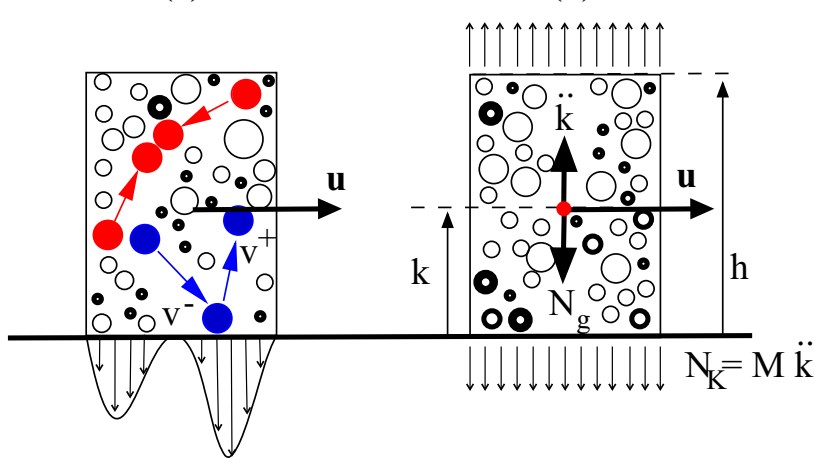

Collisional footprint

Restitution coefficients

Pre- and post

impact velocities
Dispersive acceleration

Mean normal stress

Dilatancy

Fig. 6 a Dilatancy and the dispersive pressure are often described using the concepts of granular minutiae: binary collisions, restitution coefficients, pre- and post-impact velocities, the collisional footprint, etc. $\mathbf{b}$ The result of all inelastic binary interactions is to create a mean upward acceleration $\ddot{k}$ that has a reaction at the base of the flow

study the deformation of the ensemble. Rather, the flux of random kinetic energy of the particle scattering defines - in the mean - the particle velocities and the corresponding deformation of the ensemble. Instead of considering the minutiae of the granular interactions, including the velocities of individual particles, we study the configurational energies associated with the center of mass.

We conclude that the dilatancy of the granular ensemble is constrained by the dispersive energy of the random particle movements, as well as the conversion of random kinetic energy into potential energy because of the basal boundary. These are physically limited processes. It is not possible to describe dilatancy with stress concepts because stress alone does not describe the energy usage to expand the volume. A similar approach is considered to model the collapse of the volume: Random energy is withdrawn from the granular ensemble as a whole and must not be defined by inelasticity of individual particle collisions, although this is clearly the driving process.

\subsection{Parameterization of dilatancy}

We note that the mechanics of dilatancy is best described by three bulk energy parameters: $\alpha$, the dispersion parameter that splits the shear work into heat and random scattering, $\gamma$, the dilatancy parameter that divides the random scattering energy into kinetic and potential parts, and $\beta$, the dissipation parameter controlling the decay of random kinetic energy. These parameters partition the shear power and therefore govern the magnitude of the dispersive 
acceleration in the $z$-direction. They directly control the flow configuration and therefore the frictional properties of the flow. Parameterization of the energy fluxes leads automatically to a description of different avalanche flow regimes and transition phenomena [3].

\subsection{Cohesion}

Finally, the energy formulation facilitates the introduction of additional physical processes and material properties that are important to describe further flow features of avalanches and debris flows. One such important process is cohesion, describing the "stickiness" of the particles [27]. Cohesion, also a non-directional, random process in a particle ensemble [4], can be introduced by adding the cohesive force $N_{0}$ to the work equation of dilatancy Eq. 19,

$$
W_{\mathrm{V}}=\left(N+N_{0}\right) k=\gamma \int_{0}^{t} \dot{P}(t) h(t) \mathrm{d} t .
$$

\section{References}

1. Bagnold RA (1954) Experiments on a gravity-free dispersion of large solid spheres in a Newtonian fluid under shear. Proc R Soc Lond A 225(1160):49-63

2. Bartelt P, Buser O, Platzer K (2006) Fluctuation-dissipation relations for granular snow avalanches. $\mathrm{J}$ Glaciol 52(179):631-643

3. Bartelt P, Bühler O, Buser O, Christen M, Meier L (2012) Modeling mass-dependent flow regime transitions to predict the stopping and depositional behaviour of snow avalanches. J Geophys Res 117:F01015. doi:10.1029/2010JF001957

4. Bartelt P, Vera CV, Feistl T, Christen M, Bühler Y, Buser O (2015) Modelling cohesion in snow avalanche flow. J Glaciol 61(229):837-850. doi:10.3189/2015JoG14J126

5. Bartelt P, Buser O, Vera VC, Bühler Y (2016) Configurational energy and the formation of mixed flowing/powder snow and ice avalanches. Ann Glaciol. doi:10.3189/2016AoG71A464

6. Bouchut F, Fernandez-Nieto DE, Mangeney A, Narbona-Reina G (2015) A two-phase two-layer model for fluidized granular flows with dilatancy effects. https://hal-upec-upem.archives-ouvertes. fr/hal-01161930

7. Buser O, Bartelt P (2011) Dispersive pressure and density variations in snow avalanches. J Glaciol 57(205)

8. Buser O, Bartelt P (2015) An energy-based method to calculate streamwise density variations in snow avalanches. J Glaciol. doi:10.3189/2015JoG14J054

9. George D, Iverson RM (2014) A depth-averaged debris-flow model that includes the effects of evolving dilatancy II. Numerical predictions and experimental tests. Proc R Soc A 470:20130820. doi:10.1098/rspa.2013.0820

10. Goodman RE (1999) Karl Terzaaghi. ASCE press, Reston

11. Hunt ML, Zenit R, Campell CS, Brennen CE (2002) Revisiting the 1954 suspension experiments of RA Bagnold. J Fluid Mech 452:1-24
12. Issler D, Gauer P (2008) Exploring the significance of the fluidized flow regime for avalanche hazard mapping. Ann Glaciol 49:193-198

13. Iverson RM, Logan M, LaHusen RG, Berti M (2010) The perfect debris flow? Aggregated results from 28 large-scale experiments. J Geophys Res Earth Surf 115:F03005. doi:10.1029/ 2009JF001514

14. Iverson R, George D (2014) A depth-averaged debris-flow model that includes the effects of evolving dilatancy I. Physical basis. Proc R Soc A 470:20130819. doi:10.1098/rspa.2013.0819

15. Kaitna R, Dietrich WE, Hsu L (2014) Surface slopes, velocity profiles and fluid pressure in coarse-grained debris flows saturated with water and mud. J Fluid Mech 741:277-403. doi:10. 1017/jfm.2013.675

16. Kaitna R, Palucis M, Yohannes B, Hill K, Dietrich WE (2015) Effects of coarse grain size distribution and fine particle content on pore fluid pressure and shear behavior in experimental debris flows. J Geophys Res Earth Surf. doi:10.1002/2015JF003725

17. Kowalski J, McElwaine JN (2013) Shallow two-component gravity-driven flows with vertical variation. J Fluid Mech $714: 434-462$

18. Le Roux J (2003) Can dispersive pressure cause inverse grading in grain flows? Discussion. J Sediment Res 73:333-334

19. Legros F (2002) Can dispersive pressure cause inverse grading in grain flows? J Sediment Res 72:166-170

20. Legros $F$ (2003) Can dispersive pressure cause inverse grading in grain flows? Reply. J Sediment Res 73:335-335

21. Leine R, Schweizer A, Christen M, Glover J, Bartelt P, Gerber W (2014) Simulation of rockfall trajectories with consideration of rock shape. Multibody Syst Dyn 32(2):241-271. doi:10.1007/ s11044-013-9393-4

22. McArdell BW, Bartelt P, Kowalski J (2007) Field observations of basal forces and fluid pressure in a debris flow. Geophys Res Lett 34:L07406. doi:10.1029/2006GL029183

23. Norem H, Irgens F, Schieldrop B (1987) A continuum model for calculating snow avalanche velocities. In: Salm B, Gubler H (eds) Avalanche formation, movements and effects. Proceedings of the Davos symposium, September 1986. IAHS Publication no. 162. IAHS Press, Inst. of Hydrology, Wallingford, pp 363-380

24. Platzer K, Bartelt P, Kern M (2007) Measurements of dense snow avalanche basal shear to normal stress ratios $(\mathrm{S} / \mathrm{N})$. Geophys Res Lett 34(7):L07501

25. Reynolds O (1886) Experiments showing dilatancy, a property of granular material, possibly connected with gravitation. In: Proceedings at the Royal Institution of Great Britain, Read February 12

26. Reynolds $O$ (1885) On the dilatancy of media composed of rigid particles in contact, with experimental illustrations. Philos Mag Ser 5(20):469-481

27. Rowlinson J (2002) Cohesion. Cambridge University Press, Cambridge

28. Savage S, Hutter K (1989) The motion of a finite mass of granular material down a rough incline. J Fluid Mech 199:177-215

29. Terzaghi C (1925) Principles of soil mechanics. Eng News Rec 95(19-23):25-27

30. Vera VC, Wikstroem JK, Bühler Y, Bartelt P (2015) Release temperature, snow-cover entrainment and the thermal flow regime of snow avalanches. J Glaciol. doi:10.3189/ 2015JoG14J117 